\title{
A case of abnormal sex-dimorphism for bristle number in Drosophila melanogaster
}

\author{
MARIANNE RASMUSON \\ Department of Genetics, Umeå University, S-901 87 Umeå, Sweden
}

\begin{abstract}
A line of Drosophila melanogaster with average male sternopleural bristle number higher than that of females was obtained after many generations of disruptive selection, where males with high bristle score were mated to females with low scores. This abnormal sex-dimorphism did not influence other bristle traits. Dose compensation mechanisms were not involved, and by means of chromosome substitutions it could be shown that both $\mathrm{X}$-linked and autosomal loci contributed to the sex-dimorphism.
\end{abstract}

Keywords: chromosome assay, Drosophila melanogaster, sex-dimorphism, sternopleural bristle number.

\section{Introduction}

Sex determination in Drosophila is induced by different X/A chromosomal ratios in males and females, 0.5 and 1.0, respectively. A set of regulating genes have been identified both on $\mathrm{X}$ and the autosomes which switch the development into a male or a female direction. For a recent review see Parkhurst \& Meneely (1994).

As a consequence of this mechanism genes on the $\mathrm{X}$ chromsome are hemizygous in males. To overcome the effects of this aneuploidy there is a dose compensation at work in males, acting at the level of transcription. It is known that each cell is autonomous for the compensation, that there are many regulating cis-acting sequences distributed over the $\mathrm{X}$-chromosome, and that these can act at a distance and affect transcription (Gorman \& Baker, 1994).

Dosage compensation is a way to modulate the sex differences at the cellular level. The mechanisms behind morphological and anatomical sex differences are mostly unknown, as are the limits for the flexibility of these differences. Frankham (1968, 1977), studying abdominal sternite bristle numbers in Drosophila, showed that disruptive selection with opposite directions for males and females changed the sex-dimorphism ratio $(\mathrm{SD}$ ratio $=$ male score/ female score), decreasing it when high numbers were selected for among females and low numbers among males, increasing it when the opposite selection scheme was used. Genetic variation in loci affecting the sexual dimorphism was thus present in the population. He also showed that genes influenc- ing the SD ratio for abdominal bristles were located on all the major chromosomes. Further results indicated that dose compensation for X-linked loci affecting the abdominal bristle number was present. This has also been claimed for sternopleural bristle number (A. Robertson, cited by Cock, 1964).

The genetic nature of quantitative variation according to the classical model is of many loci with infinitesimal effects (Falconer, 1989). It has been suggested that much of the quantitative variation in a trait could result from the segregation of alleles with more subtle effects at loci identified by alleles with major effects on the same character (Thompson, 1975). Concerning bristle numbers Mackay \& Langley (1990) and Lai et al. (1994) have confirmed an association between molecular variation and bristle numbers at proneural loci affecting bristle patterning. Little attention, however, has been given to the sexual dimorphism in this connection.

In the present investigation a case of abnormal, i.e. inverted, sex-dimorphism for sternopleural bristle number has been studied. To analyse the genetic mechanism reciprocal outcrosses as well as substitutions of whole chromosomes and chromosomal sections have been performed.

\section{Materials and methods}

Normally the average number of sternopleural bristles is higher for females than for males by $0.5-1$ bristle. This difference is small compared with the standard deviation for bristle number within each 
sex (Table 2). As a measure of sexual dimorphism for the character the difference between female and male average numbers, $d=\bar{x}_{0}-\bar{x}_{i}$, has been used. A negative value indicates that males on the average have more sternopleural bristles than females. The stocks used in this investigation have average bristle numbers varying from 15 to 23 and a standard deviation around 1.5. To obtain reliable results at least 20 flies of each sex were scored for each estimate of $d$. Then a $d$-value around 1 will be significant at the 0.05 level.

The variates used in the following are culture averages of female and male scored bristle numbers and $d$, the deviation between these. Stanadard deviations of the variates are computed from repetitions of the crosses or from consecutive generations of

Table 1 Mutant stocks used in crosses. Stock numbers are according to Umeå Drosophila Stock Centre list

\begin{tabular}{lc}
\hline Mutants & Stock number \\
\hline$f B$ od $c a r$ & 4500 \\
$w m f$ & 30300 \\
$y w c t^{6} m f$ & 33400 \\
$\operatorname{In}(1), r s t^{3}, y r s t^{3} c a r$ & 24300 \\
$C(1) D X, y ; n e t ; s b d^{2} ; s p a^{p o l}$ & No longer in stock \\
$s c z w^{i s} ; n e t ; s b d^{2} ; s p a^{p o l}$ & 92700 \\
$\operatorname{In}(2 L R) b w^{V 1} d p b / S M 6 a, C y d p^{l v 1} p r ;$ & \\
$T M 6 B, h D^{3} H u e / \operatorname{In}(3 R) M o, S b s r$ & 89100 \\
\hline
\end{tabular}

lines, and overall means are given with standard errors.

In experimental comparisons values are taken from simultaneous scorings and may therefore vary between experiments for the same line. The stocks used in the various crosses are listed in Table 1 . All flies were raised in bottles at $25^{\circ} \mathrm{C}$ on standard medium.

\section{Origin of the inverted dimorphism}

From each of two parallel cage populations with wild-type Drosophila melanogaster of mixed origin selection lines were started by taking in each generation the five females with lowest bristle number to be crossed to the five males with the highest bristle number. In each sex 50 individuals were scored. Simultaneously two lines from the same original population were selected for females with high and males with low bristle number with the same intensity. For 13 generations no effects of the selection could be observed. After pooling the two parallel selection lines a continued selection resulted in a line, LH, where average male bristle numbers consistently were higher than those of females. A negative $d$ was thus obtained, which remained also when selection was later relaxed. The effect of selectin in the opposite direction, high females and low males (HL), was small. Average bristle numbers were little affected by the selection but fluctuations caused by genetic drift occurred (Table 2).

Table 2 Average bristle number, variation, and sexual dimorphism $\left(d=\bar{x}_{q}-\bar{x}_{f}\right)$ for sternopleural bristles in the original populations and selected lines of Drosophila melanogaster

\begin{tabular}{|c|c|c|c|c|c|c|}
\hline & \multicolumn{4}{|c|}{ Bristle number } & & \\
\hline & \multicolumn{2}{|c|}{ Female } & \multicolumn{2}{|c|}{ Male } & & \\
\hline & Mean & SD & Mean & SD & \multicolumn{2}{|c|}{$d=\bar{x}_{q}-\bar{x}_{\hat{o}}$} \\
\hline \multicolumn{7}{|c|}{ Unselected stocks } \\
\hline I & 18.08 & 1.99 & 17.64 & 1.92 & 0.44 & \\
\hline II & 16.90 & 1.50 & 16.64 & 1.43 & 0.26 & \\
\hline \multicolumn{7}{|l|}{ Early selection } \\
\hline 13 generations & & SE & & SE & & SE \\
\hline $\mathrm{L} \times \mathrm{H} \mathrm{I}$ & 17.88 & 0.20 & 17.36 & 0.17 & 0.51 & 0.10 \\
\hline II & 18.34 & 0.13 & 17.91 & 0.14 & 0.44 & 0.10 \\
\hline $\mathrm{H} \times \mathrm{L} \mathrm{I}$ & 20.21 & 0.21 & 20.08 & 0.23 & 0.13 & 0.19 \\
\hline II & 17.65 & 0.18 & 16.93 & 0.20 & 0.72 & 0.10 \\
\hline \multicolumn{7}{|l|}{ Late selection } \\
\hline 28 generations & & & & & & \\
\hline LH & 18.29 & 0.16 & 18.68 & 0.15 & -0.40 & 0.09 \\
\hline HL & 17.94 & 0.22 & 17.32 & 0.23 & 0.62 & 0.11 \\
\hline
\end{tabular}




\section{Results}

The abnormal sexual dimorphism for the sternopleural bristle numbers has been maintained in the LH strain for many generations without selection and the condition is presumably genetically fixed. Genetic analyses have been undertaken in order to reveal factors behind this phenomenon.

The relative size of males and females was not altered. It was further shown that the dimorphism for other bristle characters was not influenced. Abdominal sternite bristles, orbital bristles and coxal bristles on the third pair of legs were scored in LH, $\mathrm{HL}$ and several wild-type stocks. Only for the sternopleural bristles did the LH line deviate from the ordinary sex-dimorphism (Fig. 1).

Indications of extrachromosomal or maternal influences were sought for in outcrosses of $\mathrm{LH}$ to three inbred wild-type lines. The $\mathrm{F}_{1} \mathrm{~s}$ were mostly intermediate as to bristle number and sexual dimorphism, and the reciprocal differences were insignificant both for male and female bristle numbers (Table 3). Only for one of the lines did $d$-values differ between reciprocal crosses, which was mostly caused by different average bristle numbers in females, and thus probably a chance effect.

\section{Substitution of $X$ chromosome and autosomes}

The $\mathrm{X}$ chromosome in the $\mathrm{LH}$ line was exchanged by outcrossing to stocks with marked $\mathrm{X}$ chromo- somes and subsequent backcrosses to the LH line. Two different substitutions were made, using $w m f$ and $y$ rst car as markers (Table 1). Four generations of backcrosses were undertaken which should make more than 96 per cent of the autosomes of $\mathrm{LH}$ origin.

The same two marker stocks were used for an exchange of autosomes, in which case the backcrosses were to the marker stock, while keeping track of the wild-type $\mathrm{X}$ chromosome.

The substitutions changed average sternopleural bristle number as well as its sexual dimorphism compared with the original LH line (Table 4).

The $\mathrm{X}$ chromosome of LH seems to carry genes increasing the number of sternopleural bristles in comparison to the mutant marker stocks. When exchanged, the bristle numbers decreased in both sexes compared to the $\mathrm{LH}$ line, but relatively less in females (about ten times the SE) than in males (15 times the SE).

Substitution of autosomes in $\mathrm{LH}$ resulted in an increase of female bristle numbers by about 5-6 SE, but the influence on male bristle number differed. When the autosomes were from the $w m f$ stock the male bristle number decreased as much as the females increased, whereas the $y$ rst car stock autosomes did not alter the male bristle number. The resulting $d$-values were negative, although less so than in the intact LH line, when the X chromosome was exchanged and when the autosomes were from the $y$ rst car stock, but positive with autosomes from the $w m f$ stock.
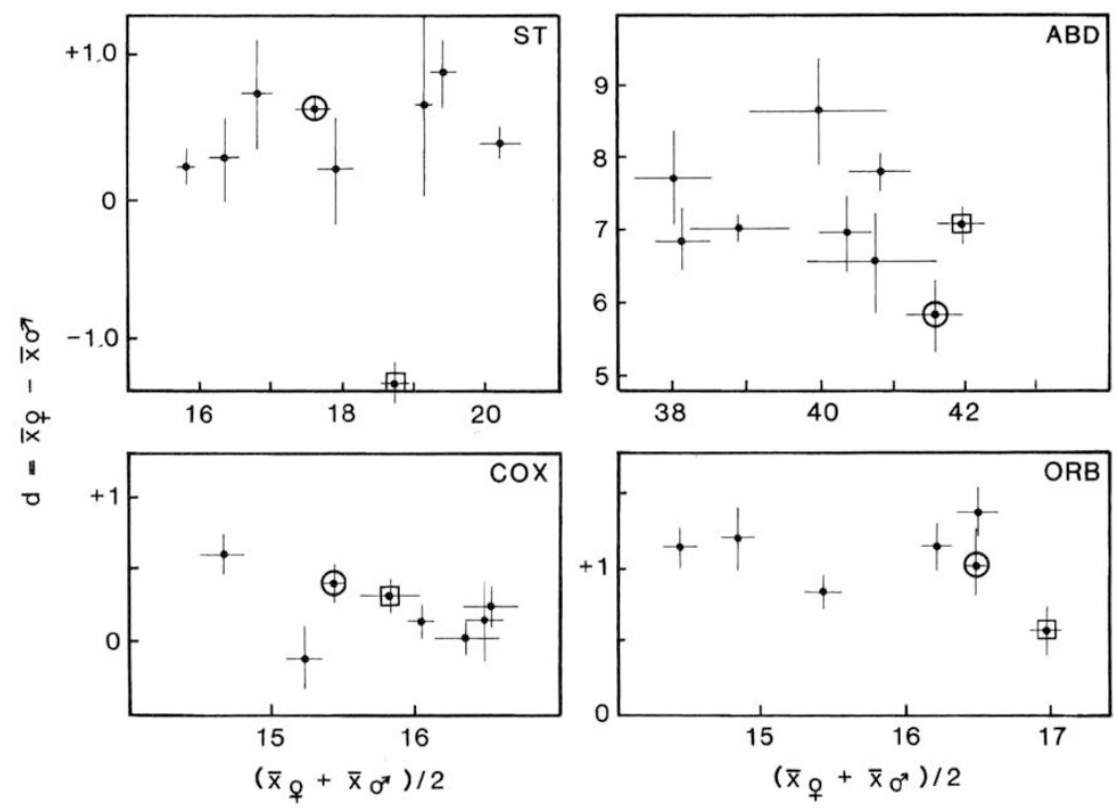

Fig. 1 Bristle numbers and sex-dimorphism for different bristle groups in line LH (square), line HL (circle), and samples of wild-type stocks of Drosophila melanogaster. Bristle numbers are given as the mean of male and female averages, sex-dimorphism as the difference $d=\bar{x}_{8}-\bar{x}_{\hat{\delta}}$. Standard errors are indicated. ST, sternopleural bristles; ABD, abdominal sternite bristles; COX, coxal bristles on third pair of legs; ORB, orbital bristles. 
Table 3 Average bristle number and sex-dimorphism, $d$, in offspring from reciprocal crosses between LH and three wild-type inbred lines of Drosophila melanogaster

\begin{tabular}{|c|c|c|c|c|c|c|}
\hline & \multicolumn{4}{|c|}{ Bristle number } & \multirow[b]{2}{*}{$d=\bar{x}_{\varphi}-\bar{x}_{\varphi}$} & \multirow[b]{2}{*}{ SE } \\
\hline & Female & $\mathrm{SE}$ & Male & SE & & \\
\hline LH & 18.46 & 0.31 & 19.05 & 0.27 & -0.59 & 0.10 \\
\hline \multicolumn{7}{|l|}{ Inbred lines } \\
\hline I & 15.42 & 0.18 & 15.31 & 0.17 & 0.11 & 0.05 \\
\hline II & 18.26 & 0.27 & 17.19 & 0.25 & 1.07 & 0.09 \\
\hline III & 17.89 & 0.25 & 18.01 & 0.33 & -0.12 & 0.21 \\
\hline \multicolumn{7}{|l|}{$\mathrm{F}_{1}$} \\
\hline $\mathrm{I} \times \mathrm{LH}$ & 16.72 & 0.50 & 16.43 & 0.51 & 0.29 & 0.10 \\
\hline $\mathrm{LH} \times \mathrm{I}$ & 17.08 & 0.80 & 17.09 & 0.62 & -0.01 & 0.25 \\
\hline II $\times$ LH & 17.55 & 0.19 & 17.00 & 0.21 & 0.55 & 0.13 \\
\hline $\mathrm{LH} \times \mathrm{II}$ & 17.96 & 0.48 & 17.41 & 0.38 & 0.54 & 0.17 \\
\hline III × LH & 18.81 & 0.35 & 18.54 & 0.21 & 0.27 & 0.25 \\
\hline $\mathrm{LH} \times \mathrm{III}$ & 18.19 & 0.35 & 18.67 & 0.20 & $-0.48^{*}$ & 0.20 \\
\hline
\end{tabular}

Average based on eight replications with standard errors (SE).

${ }^{*} P<0.05$. All other reciprocal differences are nonsignificant.

\section{Substitutions with marked autosomal chromosomes}

For a further analysis the $\mathrm{LH}$ line was outcrossed to stocks marked with dominant and recessive autosomal mutants.

1 After crosses of $\mathrm{LH}$ females to the dominant marker stock $C y / b w^{v 1} ; S b / D \quad F_{1}$ males carrying $b w^{V I}$ and $S b$ were backcrossed to $\mathrm{LH}$ females and the four different phenotypic classes in the offspring were scored for sternopleural bristles in males and females. The $\mathrm{X}$ chromosomes in both males and females were of LH origin. The male bristle excess is reduced when one or both of the large autosomes is made heterozygous, and chromosome 3 has a larger influence than chromosome 2 . The change in $d$ is caused by a higher increase in average bristle number in females than in males (Fig. 2). The $d$-values for the different phenotypic classes do not differ significantly.

2 After a cross between $\mathrm{LH}$ and a multiply marked stock with recessive markers in all autosomes $F_{1}$ males were backcrossed to females from the marker stock. This resulted in a substitution of the male $\mathrm{X}$ chromosome and one of the female ones for the $\mathrm{X}$ chromosome from the marker stock which carried the sc $z w^{i s}$ mutants. In a parallel experiment the marker stock females were of the attached-X type and carried the mutant $y$.
The offspring were grouped according to marker combinations and scored for bristle numbers in males and females. Both male and female bristle numbers decreased when the autosomes were substituted, but those in males decreased more. Because of this the male bristle number excess became smaller and vanished when all or at least both the large autosomes were derived from the marker stock. The differences between the classes were mostly nonsignificant but the trend is obvious (Fig. 3 ). In the parallel experiment the same trend was observed, although the attached-X chromosomes increased the bristle number in females to a level much higher than in males, resulting in high positive $d$-values throughout (data not shown).

\section{Partial substitution of the $X$ chromosome}

As shown, the substitution of the $\mathrm{X}$ chromosome in the LH line for that from another stock changed the sexual dimorphism of the sternopleural bristle number (Table 4). To investigate if a special part of the chromosome could be associated with the change, part of the $\mathrm{X}$ chromosome in the LH line was substituted by means of recombination.

Females from a stock with mutant genes distributed over the $\mathrm{X}$ chromosome were crossed to $\mathrm{LH}$ males. Heterozygous $F_{1}$ females were backcrossed to LH and from their male offspring different combinations of wild-type and mutant characters were 


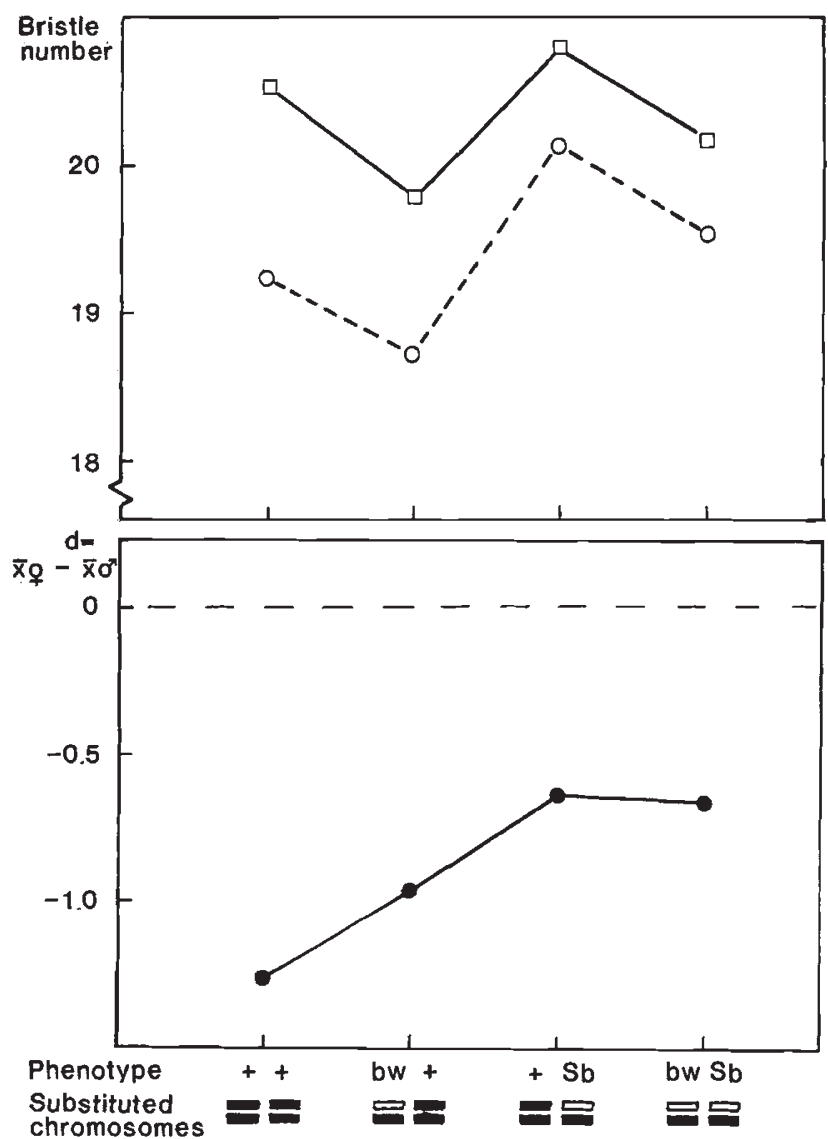

Fig. 2 Effects on sternopleural bristle number of substituting autosomes in the LH line of Drosophila melanogaster. Chromosomes from the marker stock (unfilled) carry the dominant markers $b w^{V}$ on chromosome 2 and $S b$ on chromosome 3. Each point is the mean of 21 observations. Average SE for bristle numbers is for males (squares) 0.18 , for females (circles) 0.20 , and for $d=\bar{x}_{Q}-\bar{x}_{\sigma^{*}} 0.21$.

obtained. Groups of males representing the various recombination classes by their mutant phenotypes were backcrossed to $\mathrm{LH}$ females. Offspring from these crosses were scored for bristle number. Two generations of backcrosses ought to make most of the autosomes of LH origin, as well as the sex chromosomes, except for the mutant-carrying part in one of the female $\mathrm{X}$ chromosomes. There were no significant differences in average bristle number among the classes neither for males nor for females. However, females which were heterozygous for the proximal part of the $\mathrm{X}$ chromosome, marked by the forked mutant, had higher bristle numbers than those homozygous for the $\mathrm{LH}$ chromosome in this part. The difference between forked and nonforked classes was significant. As a consequence of this the $d$-values in the $f$ classes were positive. To confirm the result the same type of substitution was performed with two different stocks with marked $\mathrm{X}$ chromosome; $y w$ ct $m f$ and $w m f$. The influence of the forked region was confirmed in both cases (Fig. 4).

\section{Discussion}

The sex-dimorphism in bristle numbers is a complex trait as it is measured as a difference between two values that are themselves quantitative traits with a considerable nongenetic variation. Both male and female changes in bristle number can influence $d$ and thus have to be considered.

Several attempts have been made to isolate the genetic factors behind variations in bristle number and to estimate the number of loci involved (Shrimpton \& Robertson, 1988). All chromosomes seem to contribute to the variation, and most investigations indicate numbers of loci counted in tens rather than in hundreds. The effect of single factors may vary from quite appreciable to very subtle. Loci with small effects are difficult to detect and estimated numbers therefore may be too low. Different bristle types are at least partly controlled by separate loci.

The sexual differences for a specific trait can be influenced by genetic variation in the following.

1 Loci that have effects on dosage compensation of the trait.

2 Loci on the $\mathrm{X}$ chromosome which in reciprocal crosses may change the average phenotype in males and thus the sex-dimorphism.

3 Autosomal loci that modulate the sex-dimorphism for the trait, either autonomously or in nonadditive interaction with other autosomal or X linked loci.

In this particular case the abnormal sex-dimorphism concerns only the sternopleural bristles. A change in some key regulator gene for bristle numbers therefore cannot be involved. Nor do the present results indicate that dosage compensation lies behind the increase of male sternopleural bristle number. This could be achieved either by control sites overcompensating bristle affecting genes on the $\mathrm{X}$ chromosome, or by the male-specific autosomal genes which have the superior control of the process. In the first case the autosomes should lack influence on the process; in the second all X-linked loci should be affected, and thus for instance also those controlling abdominal bristles.

Both male and female bristle numbers are changed by the chromosome substitutions. Numbers 

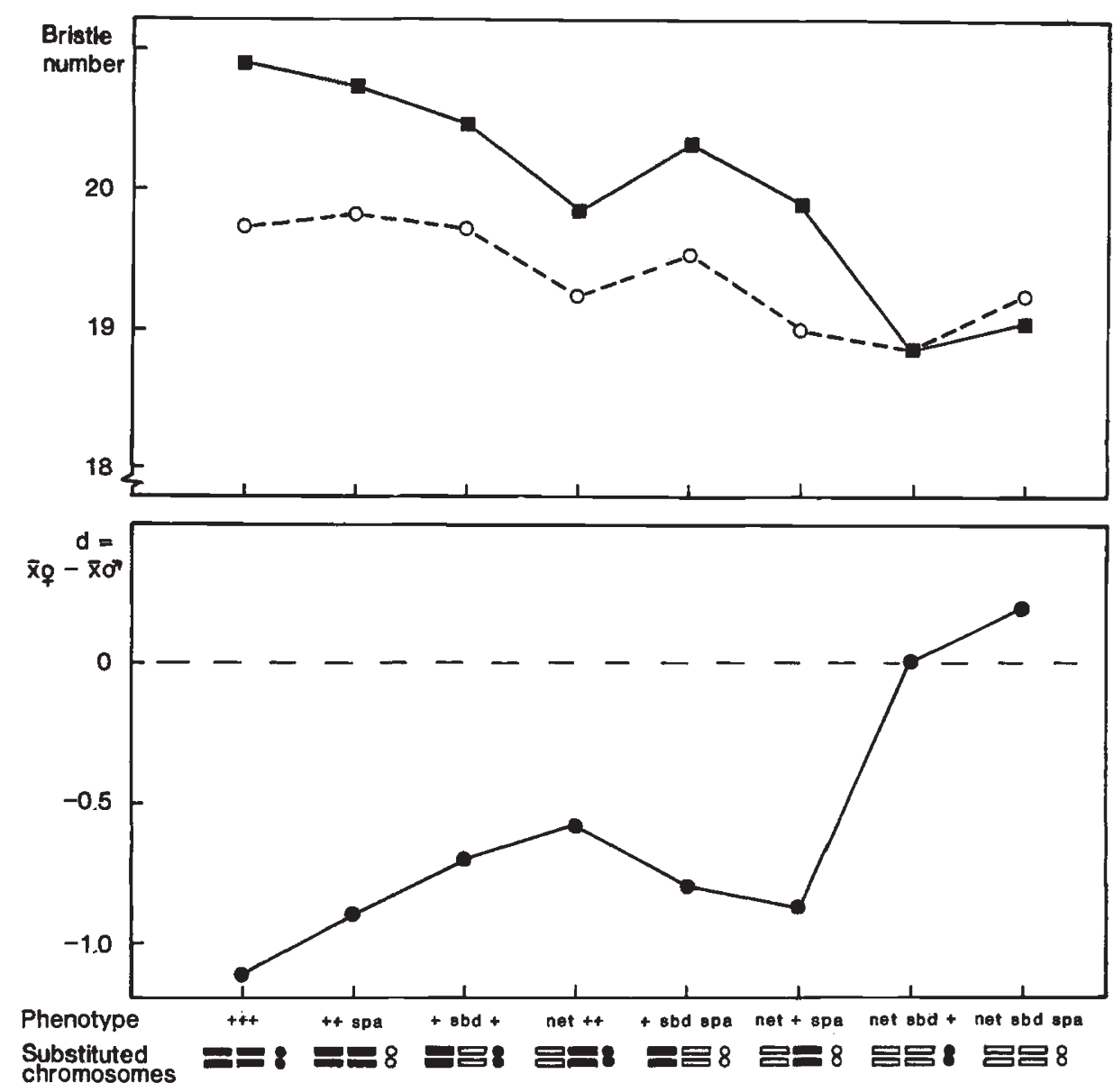

Fig. 3 Effects on sternopleural bristle number of substituting autosomes in the LH line of Drosophila melanogaster. Chromosomes from the marker stock (unfilled) carry the recessive markers net on chromosome 2, sbd on chromosome 3 and $s p a^{p o l}$ on chromosome 4. Each point is the mean of six observations. Average SE for bristle numbers is for males (squares) 0.28 , for females (circles) 0.27 , and for $d=\bar{x}_{q}-\bar{x}_{8} 0.32$.

Table 4 Effects on bristle number and sex-dimorphism of chromosomal substitutions in the LH line of Drosophila melanogaster

\begin{tabular}{|c|c|c|c|c|c|c|c|}
\hline \multirow[b]{2}{*}{ Line or stock } & \multirow[b]{2}{*}{$N$} & \multicolumn{4}{|c|}{ Bristle number } & \multirow[b]{2}{*}{$d=\bar{x}_{q}-\bar{x}_{\tilde{\sigma}}$} & \multirow[b]{2}{*}{ SE } \\
\hline & & Female & $\mathrm{SE}$ & Male & SE & & \\
\hline LH & 28 & 18.10 & 0.14 & 19.38 & 0.15 & -1.28 & 0.13 \\
\hline$w m f$ & 7 & 18.64 & 0.28 & 17.62 & 0.30 & 1.02 & 0.16 \\
\hline$y$ rst car & 15 & 16.93 & 0.13 & 16.62 & 0.14 & 0.31 & 0.11 \\
\hline \multicolumn{8}{|c|}{ LH with $X$ chromosome from } \\
\hline$w m f$ & 10 & 16.14 & 0.26 & 16.52 & 0.12 & -0.38 & 0.18 \\
\hline$y$ rst car & 24 & 16.72 & 0.17 & 17.18 & 0.17 & -0.47 & 0.12 \\
\hline \multicolumn{8}{|c|}{ LH with autosomes from } \\
\hline$w m f$ & 21 & 19.27 & 0.11 & 18.28 & 0.11 & 0.99 & 0.08 \\
\hline y rst car & 11 & 18.88 & 0.16 & 19.33 & 0.17 & -0.45 & 0.20 \\
\hline
\end{tabular}

$N$, number of cultures scored.

Average bristle numbers and $d$-values with standard errors. 

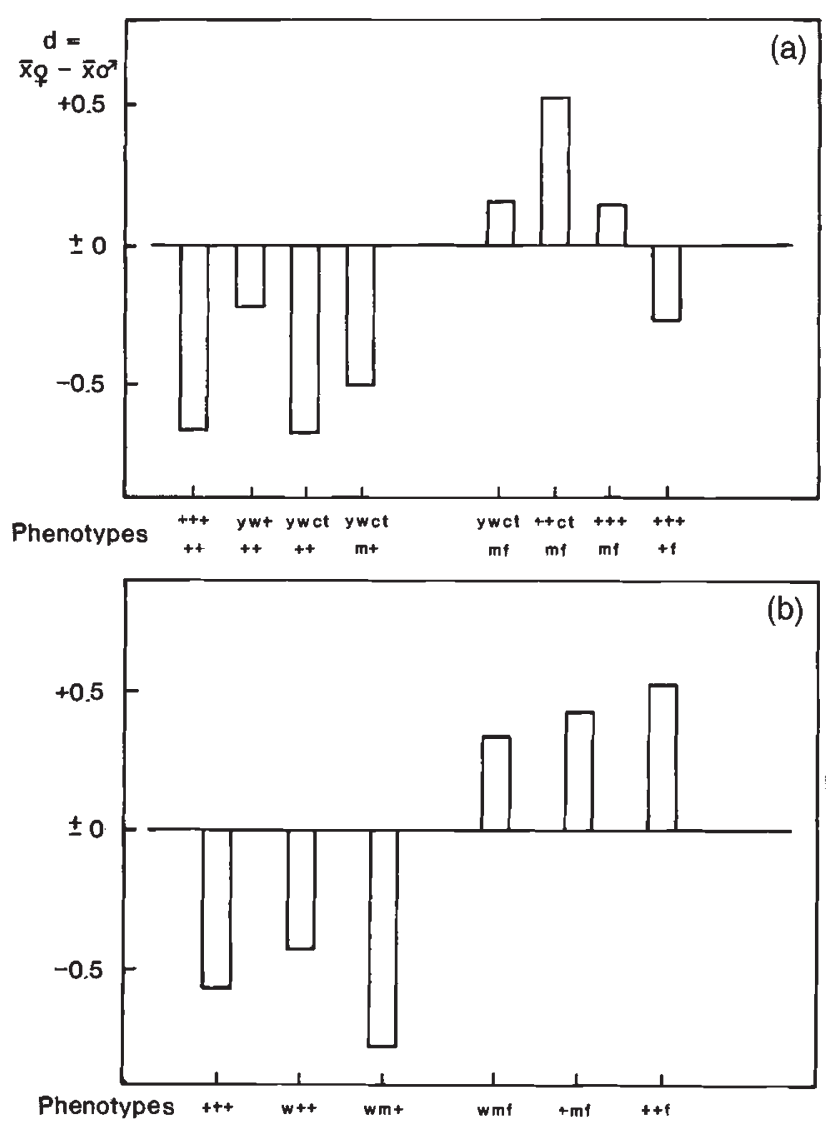

Fig. 4 Sex-dimorphism $d=\tilde{x}_{Q}-\bar{x}_{0}$ for sternopleural bristle number when part of the paternal $X$ chromosome in the LH line of Drosophila melanogaster is substituted in females. In (a) the substituted parts are marked with mutants $y w c t m f$, in (b) with $w m f$. Each $d$-value is the mean of five observations in (a), ten in (b). Average SE in (a) $=0.45$, in (b) $=0.24$.

may increase or decrease after substitutions, but usually the effect is stronger in males. Like the sternopleural bristle number itself the sex-dimorphism for this character seems to be well canalized, so that the abnormality diminishes whenever the LH genome is disturbed.

A gene in the proximal part of the $\mathrm{X}$ chromosome and autosomal loci in both the large chromosomes are indicated as affecting the sex-dimorphism in both homo- and heterozygous conditions. That genes on the $\mathrm{X}$ chromosome have differential effects on the two sexes was shown by Mackay \& Langley (1990) who analysed 36 lines with different X chromosomes but which were isogenic for all autosomes. They found that insertions in the proneural achaete-scute complex in the distal end of the $\mathrm{X}$ chromosome could explain a large part of the genetic variance in sternopleural and abdominal bristle numbers and had a differential effect on the two sexes. The fact that the scute gene also functions as one of the numerator genes $($ sis- $b$ ) in sex determination (Torres \& Sánches, 1991) might form a possible connection between bristle characters and sex-dimorphism.

The presence of another such locus in the proximal part of the $X$ chromosome is indicated by the present results, but has yet to be proven. Davies (1971), however, has located a factor for high sternopleural bristle number in the vicinity of the car locus.

Also the autosomes exercise some, apparently autonomous, influence on the sex-dimorphism. This agrees with results from the investigation by Lai et al. (1994) who analysed the effects of molecular variation on bristle numbers in another proneural locus, scabrous (sca) on chromosome 2. Among their 47 lines with different chromosome 2 origins two had an average sternopleural bristle number that was (insignificantly) higher in males than in females, and two of the polymorphic sites in the $s c a$-locus that were associated with bristle number variation had different effects in males and females.

The sex-dimorphism analysed in this investigation does not prove nor contradict the interpretation that some quantitative genetic variation can be caused by alleles at a few loci with large effects. No specific loci have been located, but a further step has been taken to highlight the problem of sex-dimorphism and relate it to the present knowledge about bristle number variation and dosage compensation.

\section{References}

COCK, A. G. 1964. Dosage compensation and sex-chromatin in non-mammals. Genet. Res., 5, 354-365.

DAVIES, R. w. 1971. The genetic relationship of two quantitative characters in Drosophila melanogaster. II. Location of the effects. Genetics, 69, 363-375.

FALCONER, D. S. 1989. Introduction to Quantitative Genetics, 3rd edn. Longman, London.

FRANKHAM, R. 1968. Sex and selection for a quantitative character in Drosophila. II. The sex dimorphism. Aust. J. Biol. Sci., 21, 1215-1223.

FRANKHAM, R. 1977. The nature of quantitative genetic variation in Drosophila. III. Mechanism of dosage compensation for sex-linked abdominal bristle polygenes. Genetics, 83, 185-191.

GORMAN, M. AND BAKER, B. s. 1994. How flies make one equal two: dosage compensation in Drosophila. Trends Genet., 10, 376-380.

LAI, C., LYMAN, R. F., LONG, A. D., LANGLEY, C. H. AND MACKAY, T. F. C. 1994. Naturally occurring variation in bristle number and DNA polymorphism at the scabrous locus of Drosophila melanogaster. Science, 266, 1697-1702. 
MACKAY, T. F. C. AND LANGLEY, C. H. 1990. Molecular and phenotypic variation in the achaete-scute region of Drosophila melanogaster. Nature, 348, 64-66.

PARKHURST, S. M. AND MENEELY, P. M. 1994. Sex determination and dosage compensation: lessons from flies and worms. Science, 264, 924-1032.

SHRIMPTON, A. E. AND ROBERTSON, A. 1988. The isolation of polygenic factors controlling bristle score in Drosophila melanogaster. I. Allocation of third chromosome sternopleural bristle effects to chromosome sections. II. Distribution of third chromosome bristle effects within chromosome sections. Genetics, 118, 437-443; 445-459. THOMPSON, J. N., Jr. 1975. Quantitative variation and gene number. Nature, 258, 665-668.

TORRES, M. AND SANCHES, L. 1991. The sisterless-b function of the Drosophila gene scute is restricted to the stage when the X:A ratio determines the activity of Sex-lethal. Development, 113, 715-722. 